\title{
Corona Current Coupling in Bipolar HVDC and Hybrid HVAC/ HVDC Overhead Lines
}

\section{Journal Article}

\section{Author(s):}

Pfeiffer, Martin D.; Hedtke, Sören (1); Franck, Christian (1)

Publication date:

2018-02

Permanent link:

https://doi.org/10.3929/ethz-b-000168489

Rights / license:

In Copyright - Non-Commercial Use Permitted

Originally published in:

IEEE Transactions on Power Delivery 33(1), https://doi.org/10.1109/tpwrd.2017.2713603 


\section{(C) 2017 IEEE.}

Personal use of this material is permitted. Permission from IEEE must be obtained for all other uses, in any current or future media, including reprinting/republishing this material for advertising or promotional purposes, creating new collective works, for resale or redistribution to servers or lists, or reuse of any copyrighted component of this work in other works."

Digital Object Identifier: 10.1109/TPWRD.2017.2713603 


\title{
Corona Current Coupling in Bipolar HVDC and Hybrid HVAC/HVDC Overhead Lines
}

\author{
Martin Pfeiffer, Sören Hedtke, and Christian M. Franck, Senior Member, IEEE
}

\begin{abstract}
This publication investigates the corona generated ion flow environment in bipolar HVDC and hybrid AC/DC transmission systems. The emphasis is on the DC ion current magnitude in the conductors. In DC conductors this constitutes the corona power loss, while in AC conductors this quantity is primarily of concern due to the risk of transformer saturation. Existing prediction methods are compared to full-scale test data from the literature and new laboratory measurements. It is shown that the bipolar HVDC and hybrid AC/DC ion flow phenomena are similar in the sense that mutual space-charge related surface field enhancements are a dominant factor in the determination of the total conductor ion currents. Furthermore, in a hybrid AC/DC environment, the existence of a net $\mathrm{DC}$ ion current stemming from the AC conductor is verified and explained. Limitations of existing prediction methods are demonstrated and recommendations for future work is given.
\end{abstract}

Keywords-HVDC, hybrid AC/DC, corona, corona losses, space charges, ion flow

\section{INTRODUCTION}

The global increase in the demand for electric energy and the connection of remote renewable energy sources is driving an expansion of transmission capacity. Converting existing HVAC transmission systems to HVDC is a widely discussed option for increasing transmission capacity in regions where the construction of new lines is difficult [1], [2].

It is estimated that a converted bipolar DC system could have more than double the capacity compared to the AC circuit that is being replaced [3]. The key factor in determining the capacity increase is the DC voltage. This in return is directly limited by the corona performance of the system.

An AC to DC conversion of a multi-circuit AC tower can constitute the replacement of all AC circuits, or only a subset thereof. The resulting tower may hold one or several bipolar HVDC systems or could also carry HVAC and HVDC systems in parallel. The latter concept has been referred to as 'hybrid HVAC/HVDC transmission'. Such a conversion will be implemented in Germany in the coming years [4].

Regardless of the nature of the conversion (partial or complete replacement of the $\mathrm{AC}$ circuits), the process raises new questions. Compared to the design of a new HVDC or hybrid HVAC/HVDC tower, a conversion will always be subject to more severe limitations with regard to the placement of conductor bundles. Separation distances between bundles (DCto-DC or AC-to-DC), for example, may have to be as low as $6 \mathrm{~m}$, given the existing tower geometry [4].

The authors are with the High Voltage Laboratory, ETH Zurich, Switzerland. Manuscript received XXXX; revised XXXX.
The aim of this paper is to investigate the corona performance of bipolar HVDC systems with small pole spacings, as well as that of hybrid HVAC/HVDC systems. Specifically, the paper will look at the corona loss (CL) characteristics of bipolar HVDC systems and the ion current coupling between HVAC and HVDC bundles in hybrid systems.

A key aspect is the use of existing numerical and empirical methods to predict the bipolar and hybrid AC/DC ion flow problem, especially for narrow pole spacings. Available literature results are analyzed and compared to calculations. Additionally, controlled laboratory studies on small scale bipolar and hybrid AC/DC test cases are presented. These studies give an insight into the fundamental space charge coupling effects that exist in bipolar DC and hybrid AC/DC systems and point out the limitations of existing methods.

\section{StATE OF THE ART}

\section{A. Bipolar Corona}

The calculation of the ion flow environment around bipolar HVDC lines has been of interest to researchers for over half a century. The first major quantitative work in this field was that of Popkov [5], who developed a model taking into account recombination and the penetration of ion of one polarity into the ionization zone of the oppositely charged conductor.

A next major step was the work by Maruvada and Janischewskyj [6] in which they presented the flux tracing method (FTM), a numerical approach of solving the ion flow problem along a finite number of field lines, which renders the 2-D problem into a discrete number of 1-D boundary value problems. Their approach makes use of the so-called Deutsch assumption, which states that ionic space charges only affect the magnitude of the electric field but not its direction.

In subsequent years, several methods were developed that waive this simplifying assumption using the finite element method (FEM), e.g. in [7], [8]. In recent years, further improvements to the stability and efficiency of numerical calculation methods have been proposed in a number of publications, including [9], [10]. In [11], the validity of the Deutsch assumption was investigated with the conclusion that for high corona intensities it can lead to significant errors.

Some of the authors of the present publication were involved in the developments of the iterative method of characteristics (IMoC) [12]. Here, the ion flow problem is solved by iterating between a FEM solver for the space charge (Poisson) field and the method of characteristics for the ion drift along discrete flux lines. No stabilization schemes are required and it is stable even for the bipolar case with wind. Compared to the FTM [6], 
the direction of the electric field is updated in each iteration (i.e. the Deutsch assumption is not made).

With regard to experimental investigations, the vast majority of studies have focused on ground level electric fields and ion current densities, including e.g. [13]-[15]. These quantities are primarily determined by the monopolar corona component of each conductor [6]. Comparing ground level data with simulation results is therefore not a viable method of verifying the model representation of the bipolar corona component. This can only be done by a comparison of the conductor CL. This publication therefore focuses on experimental sources that specifically report conductor ion currents. Notable publications that contain such data include [16]-[21].

Semi-empirical methods for CL prediction have been presented in [22], [23] and [24]. While [22], [23] derived their empirical parameters using their own data set, [24] based their equation parameters on a meta-analysis of multiple data sets from different experiments.

\section{B. Hybrid Corona}

The general performance of hybrid AC/DC lines, including electromagnetic fields and corona-related effects such as audible noise (AN) and ion currents, have been considered in several publications [1], [2], [25]-[27]. The emphasis in this publication is on DC ion currents in the $\mathrm{AC}$ and $\mathrm{DC}$ conductors, which has received comparatively little attention [2], [28], [29]. DC ion currents in DC conductors are relevant as they determine the corona losses (CL). DC ion currents in $\mathrm{AC}$ conductors are primarily of interest due to their potential of causing transformer saturation [30].

The simplest approach of solving the hybrid AC/DC ion flow is to treat AC conductors as grounded, as in [12], [31]. With regard to conductor ion currents, however, it has been demonstrated that this assumption can lead to appreciable errors in case the AC conductor is coronating [2], [29].

Accounting for $\mathrm{AC}$ corona in hybrid AC/DC ion flow simulations, has been attempted in a number of publications. The approaches can be broadly categorized as follows:

- DC equivalent simulations: representing the hybrid AC/DC case with a single DC simulation in which artificial conditions are applied to the AC conductor based on semi-empirical considerations, e.g. the 'DC equivalent voltage' method developed by EPRI in [2].

- Semi-transient simulations: Averaging a discrete number of pure DC ion flow simulations, e.g. [32].

- Transient simulations: modeling the power system frequency field component in a transient time-domain simulation, e.g. [33]-[36].

The level of complexity and computational cost of the DC equivalent simulations is lowest while that of transient simulations is highest. While [2] and [32] provided some experimental validation of conductor ion currents, all transient simulation approaches only compared their simulation results to ground level values. The impact of AC energization on ground level quantities was generally rather low. The reported difference in ground level DC electric fields between 'AC off' and 'AC on' simulations, for example, was generally in the range of roughly 10-20\% [33]-[36]. Controlled laboratory studies on a scale model of a hybrid AC/DC line have confirmed this order of magnitude [37]. The authors of [37] concluded that the AC field needs to be considered in the simulation of the DC ground level field and ion current density. They identify two mechanisms that need to be considered: the impact of $\mathrm{AC}$ energization on the corona onset voltage of the DC conductor, and the the impact of the AC field on the motion of space charges. Corona currents in the conductors were not discussed in this publication.

While the impact of $\mathrm{AC}$ energization on ground level quantities are in the range of $10-20 \%$, DC ion currents in AC conductors have been shown to differ by several $100 \%$, depending on whether AC conductors were energized or not [2]. The ability of simulation methods to predict these currents therefore deserves further investigation.

That AC conductors 'collect' a certain amount of DC ion currents when they are in proximity to a coronating DC conductor is generally established [28], [31]. That AC conductors can also be net emitters of a DC current in hybrid AC/DC energization, however, has not received much attention. This 'DC-like' behavior was first reported by EPRI in [2] and explained as follows: a nearby DC pole causes a DC field bias on the $\mathrm{AC}$ conductor and will therefore produce more space charges of one polarity than of the other. Additionally, ions of the polarity opposite to that of the DC pole will be 'pulled out of' [2] the immediate region of the AC conductor so that in the opposite half-wave they will not return to the $\mathrm{AC}$ conductor, but instead continue their drift towards the DC conductor.

In [29] laboratory measurements were presented, in which hybrid AC/DC ion flow effects were investigated by selectively wetting either the AC conductor or the DC conductor (or both) in order to control each conductor's corona behavior independently from its voltage. They were thereby able to isolate the effect of net ion production at the AC conductor, confirming EPRI's findings.

\section{METHODS}

The top view of the experimental set-up is depicted in Fig. 1. It consists of two parallel single conductors of length $6.8 \mathrm{~m}$ suspended $2 \mathrm{~m}$ above ground. The separation distance between the conductors, $D$, could be varied between approximately 1.6 and $4 \mathrm{~m}$. One conductor was connected to a DC rectifier, while the other was either connected to a rectifier or directly to an AC voltage source. Measured quantities at both lines included the voltage and the DC line currents at potential. The latter is measured via a battery powered device placed in series between the voltage sources and the conductors. It consists of an adjustable measurement resistance, an analogue to digital converter and a programmable logic device that allows the data to be accessed from a measurement computer via fibre optic cables. The device was placed inside the shielding toroid at the end of the line to prevent it from disturbing the electric field. Since only DC currents were of interest, a low-pass filter with a cut-off frequency of $1 \mathrm{~Hz}$ was installed on the measurement board. 
Stranded conductors were used with an outer diameter of $22.4 \mathrm{~mm}$ and 15 round strands in the outside layer with a diameter of $3.75 \mathrm{~mm}$. In the absence of artificial disturbances fitted to the conductors, their corona onset voltage was in the range of 190 to $240 \mathrm{kV}$ (referring to DC or AC peak voltage, other conductor grounded). The relationship between applied voltage and Laplace surface field strength is given in Table I.

In order to selectively reduce the corona onset voltage, artificial metallic disturbances in the form of sharp spikes with a circular base were attached to the conductor, as presented in [27] and shown schematically in Fig. 2 as well as in the photo inset in Fig. 3. These reduced the onset field strength to around $7 \mathrm{kV} / \mathrm{cm}$. This value was determined via tests in which the voltage of one conductor was increased in small increments while the other conductor was grounded. The first voltage at which a measurable current was detected was determined as the corona onset voltage. The corresponding onset field strength was derived based on the values given in Table I, derived using FEM. The value was found to be approximately the same for positive and negative DC, as well as AC (peak) voltages.

The disturbances were designed to roughly mimic the shape of Taylor cones formed by water on the lower side of conductors during rain [38]. It was shown in [39] that this type of metallic disturbance produces corona pulses of the same shape as those from water drops hanging from the conductor. However, certain phenomena that occur with real raindrops are not represented by the metallic disturbances. The shape of raindrops, for example, varies with the applied electrical field and a critical drop size and field is necessary to form Taylor cones [38]. Furthermore, drops behave dynamically and eject water while producing corona. Through a comparison of the data from this paper with laboratory measurements carried out with artificial rain [29], it was determined that the macroscopic corona behavior of the artificial drops is nevertheless sufficiently similar. The key advantages of the artificial disturbances are the very high reproducibility [40] and the ability to carry out measurements in a much more timeefficient manner (compared to simulated rain-fall experiments).

21 of theses disturbances with $20 \mathrm{~cm}$ separation were evenly spaced along the conductor. Tests with a higher number of disturbances per unit length showed that this only has a negligible influence on the total corona current. This is in line with observations in [39], in which a similar saturation of the current with increasing drops per unit length was observed and explained by space charge interactions between the disturbances.

Each voltage combination was held for $1 \mathrm{~min}$, during which voltages and currents were measured at $1 \mathrm{~Hz}$. A $30 \mathrm{~s}$ long exposure photograph of the conductor set-up was taken during each voltage combination. This allowed for an optical determination of the corona state of each conductor. A sample photo is shown in Fig. 3: the front conductor is fitted with disturbances and is coronating, whereas the rear one is not.

The main simulation method used in the present publication is the IMoC [12], which was briefly introduced above.

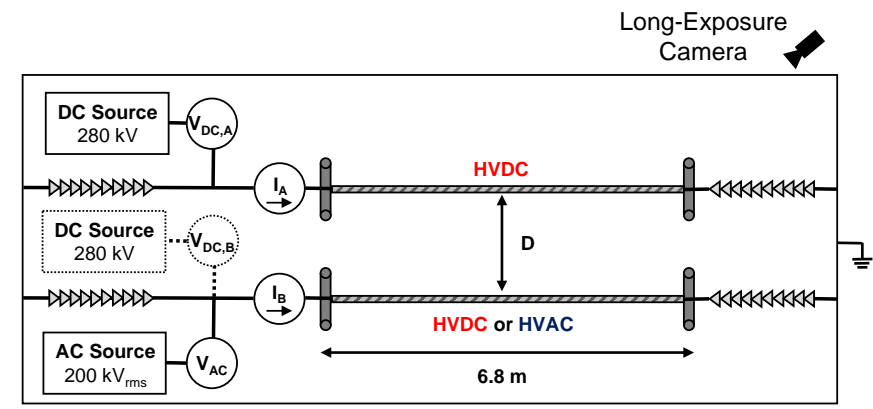

Fig. 1. Overview of Experimental Setup

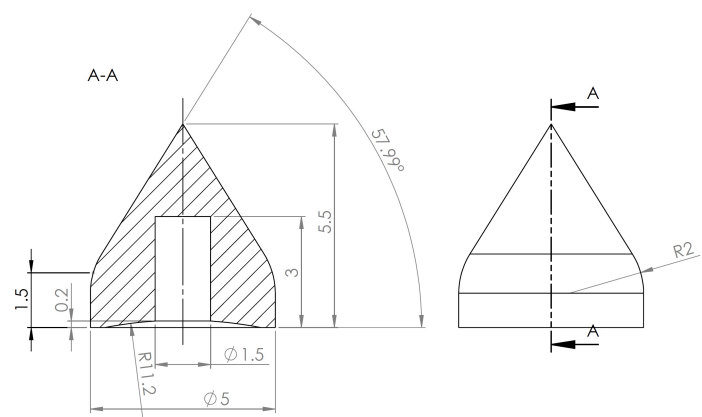

Fig. 2. Side-view drawing of artificial metallic disturbance (in mm). Base is circular.

\section{RESUlTS FOR BIPOLAR HVDC}

\section{A. Literature Comparisons - Bipolar}

Experimental results of bipolar HVDC CL have been reported in a number of publications. In this section, data from a selection of the most relevant publications are re-evaluated and compared to results from different numerical and semiempirical calculation methods.

In Fig. 4 calculation and measurement results from a bipolar test line presented in [6] are overlaid with simulation results using the IMoC [12]. Single conductors with conductor radii of $1.02 \mathrm{~cm}$ and $1.4 \mathrm{~cm}$ were tested. A conductor surface

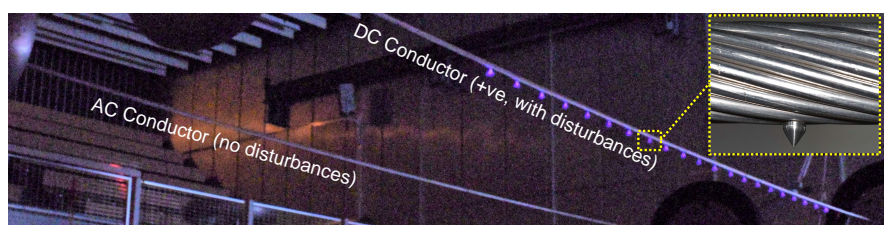

Fig. 3. Sample photo of long-term photographs used to verify the corona state of each conductor. Front conductor positive DC with disturbances (coronating), rear conductor $\mathrm{AC}$ voltage without disturbances (not coronating).

TABLE I. MAX. LAPLACE SURFACE FIELD STRENGTH ON CONDUCTORS. $100 \mathrm{KV}$ APPLIED TO ONE CONDUCTOR WHILE OPPOSITE CONDUCTOR IS AT $0 \mathrm{KV}$.

\begin{tabular}{l|l|l}
$D[\mathrm{~m}]$ & $E_{\text {max,self }}[\mathrm{kV} / \mathrm{cm}]$ & $E_{\text {max,opposite }}[\mathrm{kV} / \mathrm{cm}]$ \\
\hline 1.6 & 16.2 & 2.63 \\
4.0 & 16.4 & 0.67
\end{tabular}




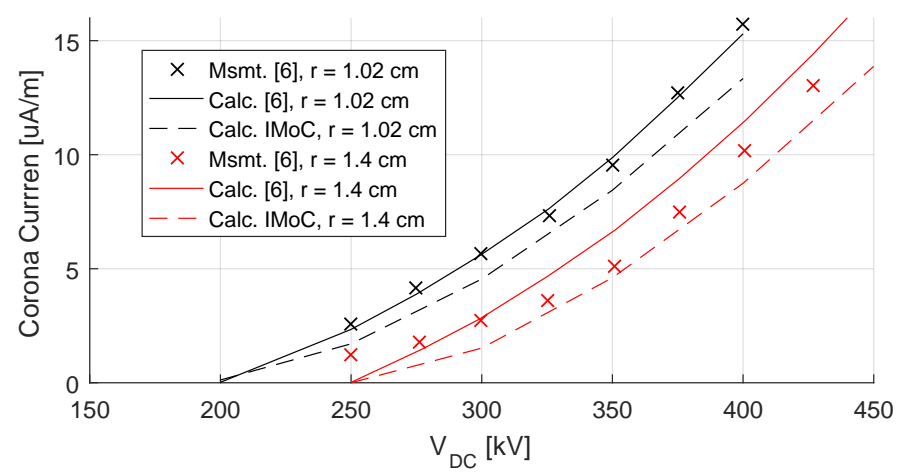

Fig. 4. Measurement and calculation of positive pole corona current for a bipolar line $(H=9.15 \mathrm{~m}, D=10.36 \mathrm{~m})$. Single conductors with radius according to legend. Assumed surface roughness $m=0.7$.

roughness value $m=0.7$ was assumed in [6], indicating fair weather conditions. The onset field strength, $E_{\text {on }}$, relates to $m$ through Peek's equation:

$$
E_{\text {on }}=m \cdot \delta \cdot E_{0} \cdot\left(1+\frac{K}{\sqrt{\delta \cdot r}}\right)
$$

where $E_{0}$ and $K$ are empirical values, $\delta$ is the relative air density and $r$ the conductor radius in $\mathrm{cm}$. In [6] for $E_{0}$ and $K$ the following value pairs were used for the positive and negative DC pole respectively: $(33.7 \mathrm{kV} / \mathrm{cm}, 0.24)$ and $(31 \mathrm{kV} / \mathrm{cm}, 0.308)$. The IMoC simulations were carried out with the same parameters. The recombination rate and ion mobility was also chosen based on [6]. The measurement data and the two calculation methods show good agreement, despite being based on fundamentally different calculation approaches.

According to (1), the $E_{\text {on }}$ of the positive pole is $29.2 \mathrm{kV} / \mathrm{cm}$, corresponding to a $V_{\text {on }}$ of around 200 and $250 \mathrm{kV}$ for the smaller and larger conductor type, respectively. In comparison to practical standards, the range of field strengths considered in [6] is extremely high. The maximum Laplace surface field strengths at $\pm 400 \mathrm{kV}$ for $r=1.01 \mathrm{~cm}$ and $r=1.4 \mathrm{~cm}$ are $58.4 \mathrm{kV} / \mathrm{cm}$ and $45.4 \mathrm{kV} / \mathrm{cm}$, respectively. Typical design limits for practical HVDC lines, however, are around $25 \mathrm{kV} / \mathrm{cm}$. The considered range therefore represents an unrealistic case, in which the conductor is likely to be in a state of significant corona even without any surface defects.

Other studies have focused on field strengths in a more practical range and have presented CL data for various weather conditions [16], [17], [20]. Rain is the most appropriate case for comparison as it shows the lowest seasonal variations and at the same time produces the highest CL [20].

Fig. 5 shows measured and computed foul weather CL values for three different test lines [16], [17], [20]. In each of these publications, average CL values for rain were explicitly or implicitly presented. Furthermore, multiple pole spacings were considered. Line heights, lengths, conductor sizes, bundle configurations and voltages varied for all presented cases.

Three different calculation approaches were used to compute the CL and overlaid onto the measurement data in Fig. 5: Firstly, a semi-empirical calculation method developed by
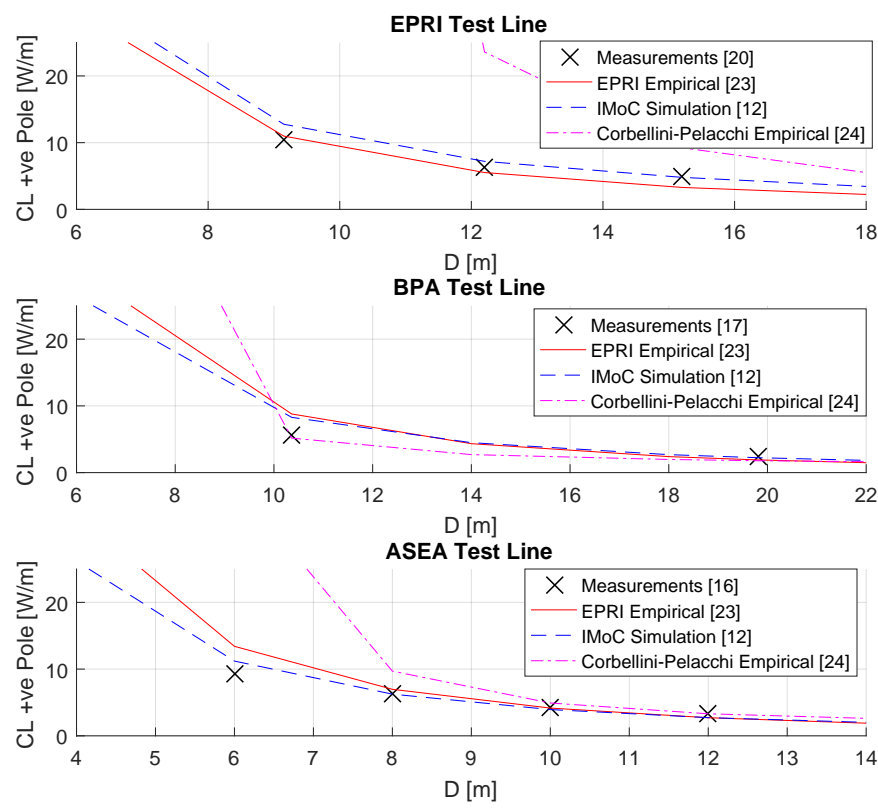

Fig. 5. Comparison of CL reported for various bipolar HVDC test lines as a function of $D$. All measurement results are mean values under rain conditions.

EPRI using the concept of degree of corona saturation, detailed in [23]. Secondly, a semi-empirical equation developed by Corbellini and Pelacchi based on a meta-analysis of a large number of full-scale outdoor test line results, detailed in [24]. And lastly, the iterative method of characteristics (IMoC) [12].

It can be seen that for all three datasets, the EPRI and IMoC computation results fit the measured results reasonably well over the considered range of $D$. The Corbellini and Pelacchi equation gives correct order-of-magnitude results for large separation distance but leads to large over-predictions of CL for smaller pole spacings.

For all three computation methods, the rate of increase of CL with decreasing $D$ is notably larger than is the case for the measured values. This range of lower spacings and the reasons for the increasing differences to the calculations are the focus of the new laboratory measurements.

\section{B. Laboratory Results - Bipolar}

Fig. 6 shows measured and simulated corona currents in conductors $\mathrm{A}$ and $\mathrm{B}$ for a bipolar energization. An extreme case with a very small pole spacing of $D=1.6 \mathrm{~m}$ is shown. $V_{\mathrm{DC}, \mathrm{A}}$ is constant at $-145 \mathrm{kV}$ while $V_{\mathrm{DC}, \mathrm{B}}$ is varied between 0 and approximately $+120 \mathrm{kV}$. Three different measurement cases are presented:

- 'v o' - In this case only conductor A is fitted with sharp disturbances (pointing downwards). Conductor B is clean and free of disturbances and does not produce corona at all (for the entire voltage range).

- ' $v$ v' - In this case both conductors are fitted with downwards pointing sharp disturbances.

- ' $><$ ' - In this case both conductors are fitted with inwards pointing sharp disturbances, i.e. the disturbances 


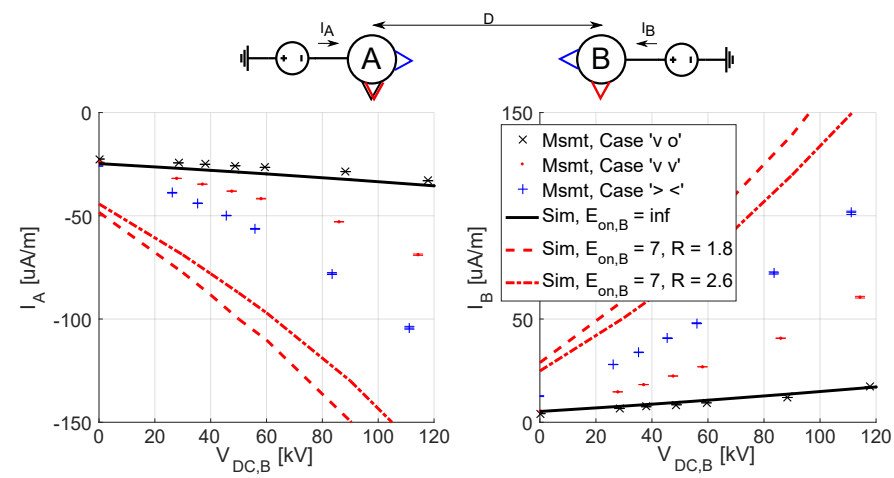

Fig. 6. Corona currents in bipolar HVDC laboratory setup as a function of $V_{\mathrm{DC}, \mathrm{B}} . V_{\mathrm{DC}, \mathrm{A}}$ constant at $-145 \mathrm{kV}$. For simulations: $E_{\mathrm{on}, \mathrm{A}}$ constant at $7 \mathrm{kV} / \mathrm{cm}$; $E_{\mathrm{on}, \mathrm{B}}$ according to legend (in $\mathrm{kV} / \mathrm{cm}$ ). $R$ in $1 \times 10^{-12} \mathrm{~m}^{3} \mathrm{~s}^{-1} . D=1.6 \mathrm{~m}$.

are pointing towards the opposite conductor.

The ' $v$ o' case is bipolar in terms of the electrostatic (Laplace) field, but only monopolar in terms of the spacecharge enhanced (Poisson) field. That is, negative ions drift from conductor A to conductor B (and ground) but there is no production of positive ions anywhere in the domain. For $V_{\mathrm{DC}, \mathrm{B}}=0 \mathrm{kV}$, the corona current produced by conductor $\mathrm{A}$ for this case is around $-22.5 \mu \mathrm{A} / \mathrm{m}$, of which around $4.2 \mu \mathrm{A} / \mathrm{m}$ $(18.7 \%)$ are collected by conductor B. With increasing $V_{\mathrm{DC}, \mathrm{B}}$, the corona current produced by conductor $\mathrm{A}$ increases steadily and reaches $-33.0 \mu \mathrm{A} / \mathrm{m}$ for $V_{\mathrm{DC}, \mathrm{B}}=118 \mathrm{kV}$, of which $17.1 \mu \mathrm{A} / \mathrm{m}(51.8 \%)$ are collected by conductor B.

Case ' $\mathrm{v}$ o' is simulated with the IMoC by applying the experimentally determined $E_{\text {on }}$ of $7 \mathrm{kV} / \mathrm{cm}$ to conductor A, while setting that of conductor B to infinity (black lines). It can be seen that the simulated corona currents in both conductors closely match the measured values for this case.

In the 'v v' case, both conductors have a reduced $E_{\text {on }}$ and readily produce corona. This mirrors the behavior of an actual bipolar HVDC system under rain conditions. For $V_{\mathrm{DC}, \mathrm{B}}=0 \mathrm{kV}, I_{\mathrm{A}}$ is nearly identical to that of case ' $\mathrm{v} \mathrm{o}$ '. With increasing $V_{\mathrm{DC}, \mathrm{B}}$, however, the currents in both conductors significantly exceed the values determined for the ' $v$ o' case. Optical corona observations confirm that discharges are observed on conductor $\mathrm{B}$ already at $V_{\mathrm{DC}, \mathrm{B}}=27 \mathrm{kV}$, leading to a bipolar space charge environment. While the Laplace field strength of conductor $\mathrm{B}$ at $V_{\mathrm{DC}, \mathrm{B}}=27 \mathrm{kV}$ is still below the onset value of $7 \mathrm{kV} / \mathrm{cm}$, the space charge enhanced field evidently already exceeds the critical value. At $V_{\mathrm{DC}, \mathrm{B}}=114 \mathrm{kV}$, corona currents in conductors $\mathrm{A}$ and $\mathrm{B}$ are $-68.8 \mu \mathrm{A} / \mathrm{m}$ and $60.6 \mu \mathrm{A} / \mathrm{m}$, respectively.

Interestingly, simulating the 'v v' case by applying an $E_{\text {on }}$ of $7 \mathrm{kV} / \mathrm{cm}$ to both conductors, leads to a significant overprediction of the corona currents in both conductors. This is true for a broad range of assumed recombination rates (red lines). For a larger separation distance of $D=4 \mathrm{~m}$, this overprediction is lower but still significant (see Table II).

In the ' $><$ ' case, higher currents are measured in both conductors compared to the ' $v$ v' case, indicating that the direction of corona disturbances affects mutual corona coupling.
TABLE II. MEASURED AND SIMULATED VALUES OF $I_{\mathrm{A}}$ AND $I_{\mathrm{B}}$ FOR TWO DIFFERENT POLE SEPARATIONS. $V_{\mathrm{DC}, \mathrm{A}}=-145 \mathrm{kV}$, $V_{\mathrm{DC}, \mathrm{B}}=117 \mathrm{kV}$, CASE 'V V'. BIPOLAR ENERGIZATION.

\begin{tabular}{l|lll|lll}
$\begin{array}{l}D \\
{[\mathrm{~m}]}\end{array}$ & $\begin{array}{l}I_{\mathrm{A}, \mathrm{meas}} \\
{[\mu \mathrm{A} / \mathrm{m}]}\end{array}$ & $\begin{array}{l}I_{\mathrm{A}, \mathrm{sim}} \\
{[\mu \mathrm{A} / \mathrm{m}]}\end{array}$ & $\begin{array}{l}\text { Deviation } \\
{[\%]}\end{array}$ & $\begin{array}{l}I_{\mathrm{B}, \text { meas }} \\
{[\mu \mathrm{A} / \mathrm{m}]}\end{array}$ & $\begin{array}{l}I_{\mathrm{B}, \mathrm{sim}} \\
{[\mu \mathrm{A} / \mathrm{m}]}\end{array}$ & $\begin{array}{l}\text { Deviation } \\
{[\%]}\end{array}$ \\
\hline 1.6 & -68.8 & -189 & 174 & 60.6 & 176 & 190 \\
4.0 & -34.4 & -51.1 & 49 & 19.1 & 40.8 & 113
\end{tabular}

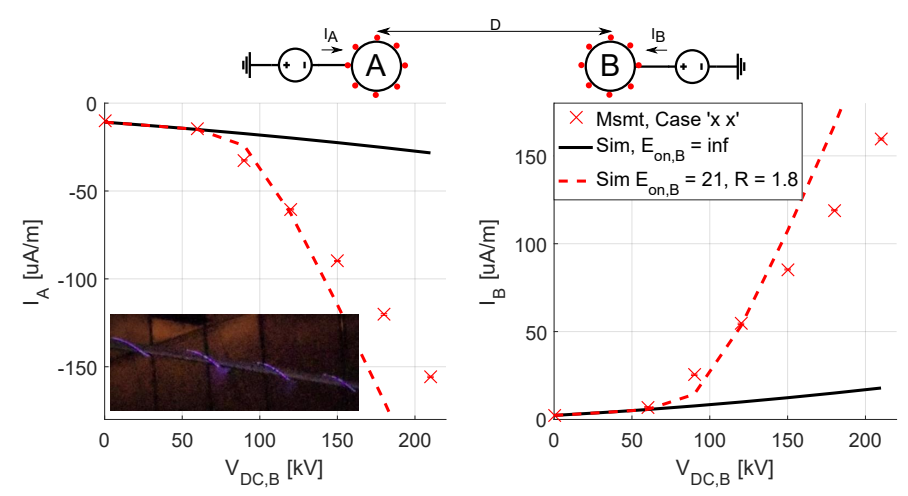

Fig. 7. Corona currents in bipolar HVDC lab setup as a function of $V_{\mathrm{DC}, \mathrm{B}}$ for ball-wire disturbance. $V_{\mathrm{DC}, \mathrm{A}}$ constant at $-180 \mathrm{kV}$. For simulations: $E_{\mathrm{on}, \mathrm{A}}$ constant at $21 \mathrm{kV} / \mathrm{cm} ; E_{\mathrm{on}, \mathrm{B}}$ according to legend (in $\mathrm{kV} / \mathrm{cm}$ ). $R$ in $1 \times 10^{-12} \mathrm{~m}^{3} \mathrm{~s}^{-1} . D=1.6 \mathrm{~m}$

Another experimental case was studied in which the charge injection around the conductor is closer to the model representation in the IMoC. In Fig. 7 results using a special ball-wire type disturbance are shown. This consists of a ballwire being helically wrapped along each conductor, leading to a reduced corona onset around the entire circumference of the conductor (c.f. lower left inset in Fig. 7). This case is labeled as ' $\mathrm{x}$ x' (ball-wire disturbances on both conductors). It bears closer resemblance to the model representation in the IMoC simulation, in which the specified $E_{\text {on }}$ is applied homogeneously to the entire conductor circumference. The experimentally determined $E_{\text {on }}$ with this type of disturbance is $21 \mathrm{kV} / \mathrm{cm}$. Using this value, the simulation is generally in good agreement with the measurements.

As an aid to the following discussion, Figure 8 shows the simulated distribution of the electric potential, the electric field and the space charge density along a horizontal line between conductor A and B (lower three plots). The plot on top shows a 2D close-up view of the two conductors overlaid with field lines and space charge density distributions. The distribution of $V$ shows that the space charges extend the potential further into the inter-electrode region. The distribution of $E$ shows that in the Poisson case, the surface fields have converged to $E_{\text {on }}$, congruent with the model assumptions. Furthermore, space charges in the inter-electrode region lead to a higher but also more homogeneous electric field strength in the centre between the conductors. Lastly, the distribution of $\rho$ shows that in the steady state solution there is a bipolar space charge density at the surfaces of both conductors. It is dominated by space charges of the same polarity but, in the presented case, the total space charge density also includes about $15 \%$ ions of the opposite polarity. 


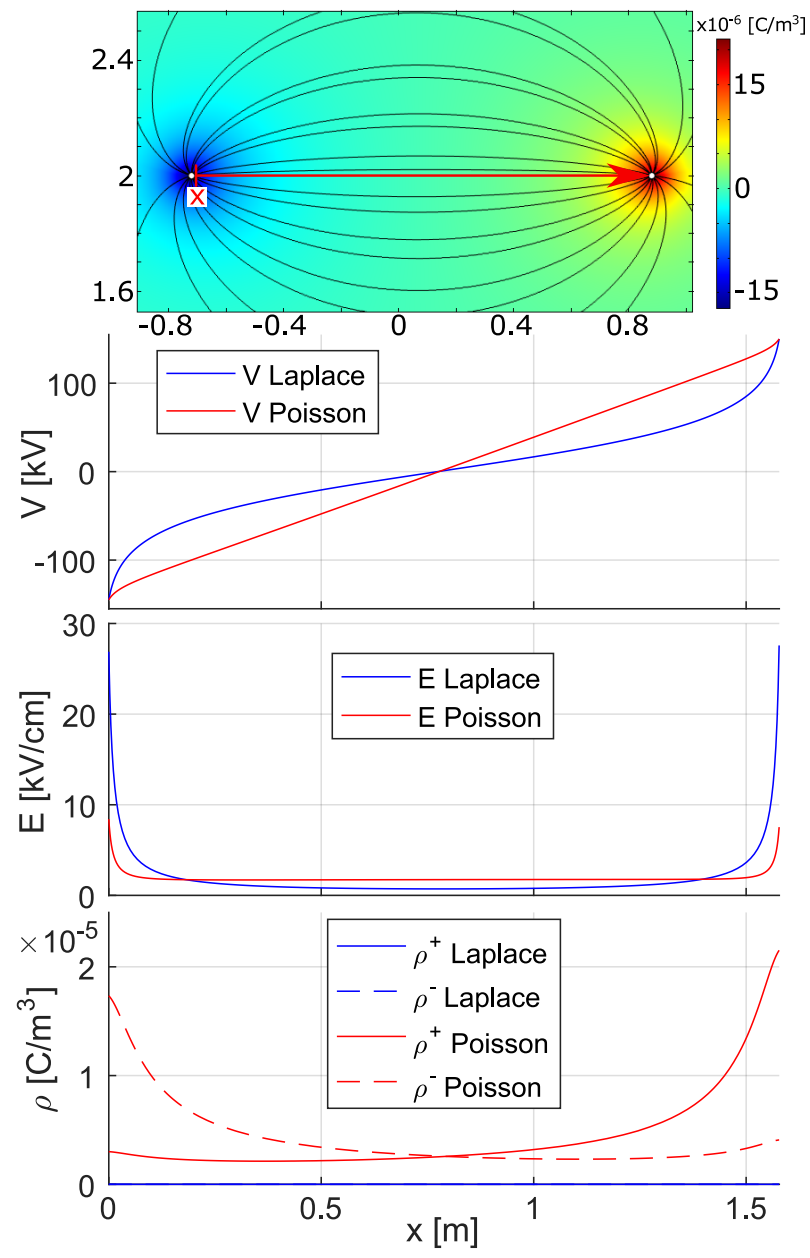

Fig. 8. Simulated electric potential, electric field strength and space charge density distribution for: $V_{\mathrm{DC}, \mathrm{A}}=-145 \mathrm{kV}, V_{\mathrm{DC}, \mathrm{B}}=150 \mathrm{kV}, E_{\mathrm{on}, \mathrm{A}}$ and $E_{\mathrm{on}, \mathrm{B}}$ both $7 \mathrm{kV} / \mathrm{cm}$ (Poisson case), $\mathrm{D}=1.6 \mathrm{~m}$. Color in top plot: $\rho^{+}-\rho^{-}$.

\section{Discussion of Bipolar Results}

The comparison of two numerical methods (the FTM and the IMoC) with fair weather measurement data showed a good general agreement between the two methods and the measurement data. In the considered case (Fig. 4), the surface electric field on the conductor was significantly above practical limits and the conductor is likely to have been in a state of 'wire corona'. This term was coined by Hara et al. [41] to describe corona that is caused by high field strength on the conductor material itself, rather than through external field disturbances (water, dirt, etc). For this case it is reasonable to assume that corona production is, on average, uniformly distributed around the circumference of the conductor (for a single conductor). This behavior corresponds to the way the charge injection is modeled in the two numerical methods.

More realistic measurement data comes from test lines in which the conductor field strength was in a practical range such as those presented in Fig. 5. Focusing on the foul weather case, the data was compared to two semi-empirical methods and IMoC simulations for various pole spacings, $D$. The approach presented by EPRI, as well as the IMoC simulation, match the measured data fairly well over wide ranges of $D$. The close agreement between EPRI's approach and the IMoC simulations is quite remarkable, given their very different nature. The empirical equation by Corbellini and Pelacchi [24] only fits the measured data for high values of $D$. The parameters for their equation were derived based on the analysis of a large number of experimental data sets, including a significant amount of data for larger pole spacings and a higher number of subconductors per bundle. Based on the presented results, for narrow pole spacings and sub-conductor numbers of 2 or 1 the equation appears unsuitable.

The results in Fig. 5 indicate that the prediction methods all show a rate of increase of CL with decreasing $D$ that is higher than the experimentally determined rate. However, only one of the data sets [16] included data for values of $D<$ $9 \mathrm{~m}$, so a general conclusion cannot be drawn. As stated in the introduction, one of the key objectives of this paper is to investigate CL prediction methods for converted HVDC or hybrid HVAC/HVDC tower geometries, in which $D$ may be as low as $6 \mathrm{~m}$ [4]. Therefore laboratory tests were carried out to compare computations with measurements for significantly lower values of $D$.

In a first experiment, the prediction accuracy of the IMoC method was investigated for a case in which only one of the bipoles produces corona (the ' $\mathrm{v}$ o' case). For this case, an excellent match between simulation and measurements was observed. However, for the case in which both conductors produce corona (the ' $\mathrm{v} v$ ' case), the simulation significantly over-predicts the measured values. The extent of this overprediction was shown to increase significantly with a reduction in $D$ (c.f. Table II). The fact that the ' $\mathrm{v}$ o' case is modeled correctly, while the ' $\mathrm{v} v$ ' case is not, indicates that the modeling deficiency lies within those model aspects that are unique to the bipolar problem.

One such modeling aspect is ionic recombination. The simulation results' sensitivity to variations in the assumed recombination rate $R$ was investigated in Fig. 6 and was shown to be minor for reasonable ranges of $R$. Furthermore, excellent agreement with measurement data for larger pole separations (c.f. Figs. 4 and 5) makes it unlikely that a fundamental error with respect to ionic recombination exists. The over-prediction is therefore unlikely to be caused by modeling assumptions related to recombination.

A second aspect that is unique to the bipolar problem is the influence of Poisson surface field enhancements due to space charges from one conductor on the corona production of the opposite conductor. In a horizontal bipolar HVDC arrangement, the Poisson surface field enhancement due to ions drifting over from the opposite pole is strongest on the conductor side that is facing towards the other conductor (i.e. 'inwards'). In the simulation, the entire conductor boundary is treated as a surface on which the $E_{\text {on }}$ is set to the same value. For foul weather conditions, this is set to a low value. Thus, in the simulation, the surface section that is subject to the largest Poisson field enhancement due to space charges from the opposite pole (i.e. the 'inwards' section) coincides with an area of low $E_{\mathrm{on}}$. The Poisson field enhancement is 
not only limited to the 'inwards' region but also enhances the field all around the conductor. The simulation thus exhibits a fairly homogeneous space charge injection around the entire circumference (c.f. Figure 8).

In reality, however, during steady rain, corona originates nearly exclusively from suspended drops on the lower side of the conductor [38], similar to the 'v v' case. Also, the area with the strongest Poisson field enhancement ('inwards') does not coincide with the area with a low $E_{\text {on }}$ ('downwards'). Additionally, the directional emission of ions can lead to a space charge distribution on the opposite conductor that deviates from the model representation.

The hypothesis that this mismatch is responsible for the large over-prediction for the bipolar ' $v$ v' case, is supported by the counter-example presented in Fig. 7. Using the ball-wires (case ' $\mathrm{x} x$ '), where corona disturbances exist uniformly around the entire conductor circumference, a close match between simulation and measurements was observed. The fact that the simulation models this case accurately, supports the above hypothesis. To fully prove this hypothesis, an adjustment in the modeling of the charge injection in a way that allows a strong directional bias, would constitute the next step.

The deviation between the simulation and the ' $i$ ' case is smaller compared to the ' $v \mathrm{v}$ ' case, but a significant overprediction remains. This may at first appear to contradict the explanation above, since in this experiment the area with the strongest Poisson field enhancement coincides with the location of the disturbance. However, in the simulation, the Poisson field enhancement affects a significantly larger portion of the conductor circumference, compared to the relatively narrow angular section in which there is a reduced corona onset in the experiment. It is therefore still plausible that the over-prediction in the simulation comes from the fact that a low $E_{\text {on }}$ is applied to the entire circumference, and not only a section thereof.

The fact that the mismatch between simulation and measurements for bipolar CL is significantly larger for small values of $D$ requires further investigation. One possible explanation is related to ionic diffusion, which is neglected in the simulation models. For larger separation distances, diffusion could lead to a more homogeneous increase of the surface field of the opposite conductor, including the lower conductor side. Assuming that corona sources are primarily on the lower conductor side (as is the case in rain), this could lead to a relative increase in CL (compared to simulation results) for large distances compared to small ones and could explain the behavior.

To summarize, the results in this section show that the typical representation of conductor surfaces as perfect circles with a constant $E_{\text {on }}$ around the circumference may, under certain circumstances, lead to significant CL prediction errors in bipolar HVDC systems. When corona sources are concentrated on the lower conductor side, as is the case in rain, simulations significantly over-predict CL if the pole spacing is small. To what extent practical pole spacings and bundle conductors are affected by this remains open for further investigations.

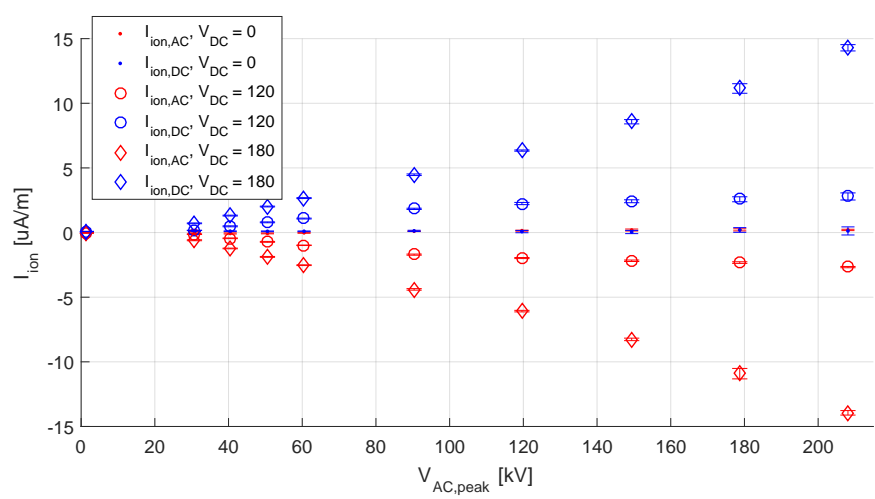

Fig. 9. DC ion current in the $\mathrm{AC}$ and $\mathrm{DC}$ conductor as a function of $\mathrm{AC}$ voltage. DC conductor: no spikes, $V_{\mathrm{DC}}$ according to legend in $\mathrm{kV}$. AC conductor: spikes, variable voltage. $\mathrm{D}=1.6 \mathrm{~m}$.

\section{HYBRID RESULTS}

\section{A. Laboratory Results}

Fig. 9 shows the DC ion current in the AC and DC conductors for a hybrid energization scenario (defined as $I_{\text {Ion,AC }}$ and $I_{\text {Ion,DC, }}$ respectively). In the shown case, the AC conductor is fitted with downward facing spikes, while the $\mathrm{DC}$ conductor is clean and free of disturbances. I.e. only the $\mathrm{AC}$ conductor produces corona, while the DC conductor only contributes a DC Laplace field. The absence of corona on the DC conductor in the presented measurements was verified with long-exposure photography and is also confirmed by the fact that at $V_{\mathrm{AC} \text {,peak }}=0 \mathrm{kV}$, the measured currents are zero regardless of $V_{\mathrm{DC}}$.

The solid lines show that there is no DC ion current in either conductor for $V_{\mathrm{DC}}=0 \mathrm{kV}$, although the $\mathrm{AC}$ conductor produces corona starting from around $V_{\mathrm{AC} \text {,peak }}=50 \mathrm{kV}$ (confirmed by long-exposure photography). Once a DC voltage is applied, however, an equal and opposite DC current is measured in both conductors. The minimum $\mathrm{AC}$ voltage required for this current to be observed depends on the applied DC voltage, as the $\mathrm{DC}$ field bias lowers the AC corona onset voltage. The current increases with increasing $\mathrm{AC}$ and $\mathrm{DC}$ voltage.

These results are a clear demonstration of the "DC-like behavior" of AC conductors, discussed in Section II. It is evident that the DC ion currents only exist due to corona on the surface of the AC conductor. The fact that the magnitude of the currents is identical shows that the net DC current from the $\mathrm{AC}$ conductor flows entirely to the $\mathrm{DC}$ conductor.

Fig. 10 shows $I_{\text {Ion,AC }}$ for multiple DC and AC voltages and different conductor separation distances. All data in this Figure is derived from measurements with downwards facing spikes on both conductors. The voltage ranges have been chosen such that the conductor surface field strengths are within a range of practical relevance (ranging from around 20 to $34 \mathrm{kV} / \mathrm{cm}$ ).

Comparing the measurement values for $V_{A C, \text { peak }}=0 \mathrm{kV}$ with those for $V_{A C \text {, peak }} \neq 0 \mathrm{kV}$ shows clearly that the $\mathrm{AC}$ voltage has a major impact on $I_{\mathrm{Ion}, \mathrm{AC}}$.

The red curves show ion flow simulation results based on 

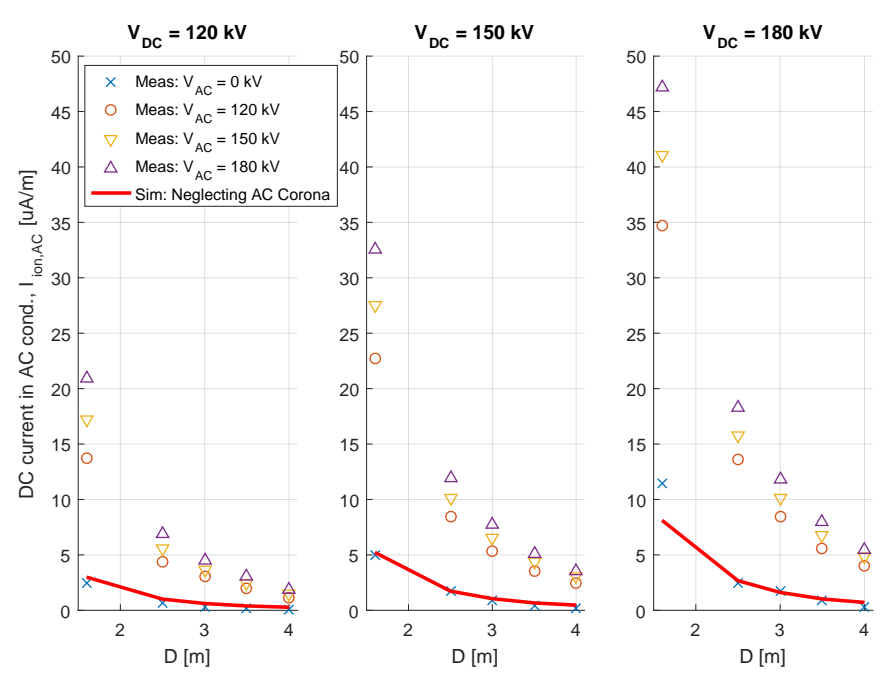

Fig. 10. DC ion current in AC conductor as a function of separation distance for different combinations of AC and DC voltages. Spikes on both conductors in all cases.

the IMoC method using the assumption of a grounded AC conductor, which are in excellent agreement with the measured values for $V_{A C, \text { peak }}=0 \mathrm{kV}$. However, for $V_{A C, \text { peak }} \neq 0 \mathrm{kV}$, clearly this assumption would lead to large under-predictions

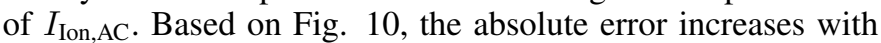
decreasing $D$, whereas the relative error is somewhat higher towards larger values of $D$.

\section{B. Results from Full-Scale Outdoor Test Lines}

Results from full-scale hybrid test lines are unfortunately very scarce. Only two full scale hybrid AC/DC test lines could be found in the literature in which the DC ion current in an $\mathrm{AC}$ conductor was reported [2], [4]. Fortunately, both sources include data values that directly contrast the DC ion currents in $\mathrm{AC}$ and $\mathrm{DC}$ conductors with and without $\mathrm{AC}$ energization (see Table III).

The data of the two experiments is not readily comparable since weather conditions, bundle types, spacing between AC and DC conductor and voltages all vary. What is of primary interest in the presented data is the relative difference in the ion currents for the case $V_{A C}=0$ and $V_{A C} \neq 0$.

The data from both experiments confirm that the AC voltage plays an important role in determining the DC ion currents. In particular for the DC ion current in the AC conductor $\left(I_{\mathrm{Ion}, \mathrm{AC}}\right)$, the difference between $V_{A C}=0$ and $V_{A C} \neq 0$ case is critical (c.f. Table III). In the measurements of [2] $I_{\text {Ion,AC }}$ for the $V_{A C} \neq 0$ case is approximately 7-10 times larger compared to the $V_{A C}=0$ case. The data from [4] indicates a factor of approximately 2 .

\section{Discussion of Hybrid Results}

The results in Figs. 9 through 10 have clearly demonstrated the "DC like behavior" of coronating AC conductors that has previously been described by [2]. While academic in nature,
TABLE III. DC ION CURRENTS IN AC AND DC CONDUCTOR FOR TWO DIFFERENT HYBRID AC/DC FULL-SCALE TEST LINES. ' $\mathrm{X}$ ' = NO DATA.

\begin{tabular}{l|l|ll|ll} 
Ref. & Weather & $\begin{array}{l}V_{A C} \\
{\left[\mathrm{kV}_{\text {rms }}\right]}\end{array}$ & $\begin{array}{l}V_{D C} \\
{[\mathrm{kV}]}\end{array}$ & $\begin{array}{l}I_{\text {Ion, AC }} \\
{[\mu \mathrm{A} / \mathrm{m}]}\end{array}$ & $\begin{array}{l}I_{\text {Ion,DC }} \\
{[\mu \mathrm{A} / \mathrm{m}]}\end{array}$ \\
\hline$[2]$ & Dry & 0 & 540 & -0.07 & 9.47 \\
{$[2]$} & Dry & 800 & 540 & -0.71 & 13.5 \\
\hline$[2]$ & Dry & 0 & -540 & 0.15 & -10.0 \\
{$[2]$} & Dry & 800 & -540 & 1.12 & -12.7 \\
\hline$[4]$ & Rain & 0 & 450 & -5.5 & $\mathrm{x}$ \\
{$[4]$} & Rain & 245 & 450 & -10.3 & $\mathrm{x}$
\end{tabular}

the controlled laboratory studies newly presented here allow a more in-depth view into the processes involved in hybrid AC/DC corona. By selectively controlling the corona state of the $\mathrm{AC}$ and $\mathrm{DC}$ conductors through artificial disturbances, for example, it was shown that a DC Laplace field is sufficient to create a $\mathrm{DC}$ ion current in a coronating $\mathrm{AC}$ conductor.

If both conductors (AC and DC) produce corona, the result is a bipolar space charge environment similar to that in a bipolar HVDC system. The AC conductor in this case not only collects a DC current, but also emits one. While the time-average of this current has a DC component, it is in fact a pulsed removal of space charges during one of the AC half cycles. The net ion drift from the AC conductor to the DC conductor enhances the surface field strength at the DC conductor leading to more corona at the DC conductor. Likewise, the drift of ions from the $\mathrm{DC}$ conductor to the AC conductor increase the surface field strength at the AC conductor (in the half-wave opposite to that of the DC polarity), enhancing corona production there. That is, there is a mutual space charge related corona enhancement similar to the behavior in a bipolar HVDC system.

That the impact of AC corona on the DC ion current coupling between AC and DC conductors is of practical relevance has been shown using full-scale test data from two separate outdoor experiments [2], [4]. Unfortunately, the available data is limited to a few data points. More data from outdoor test lines is required to develop or validate reliable models for the prediction of DC ion current coupling in hybrid AC/DC environments. Such data should include long-term statistical observations for various environmental conditions, as well as a broad variation of geometric and electrical parameters.

\section{CONCLUSION}

\section{A. Bipolar Corona}

- Different empirical and numerical methods for the prediction of bipolar CL were compared to measurement data from full-scale test lines. Two of the considered methods showed good agreement while one largely overpredicted the measured values. A general trend towards an over-prediction of CL for small pole separations was identified.

- This trend was confirmed in laboratory studies. For the case of localized disturbances on the lower conductor side (as during foul weather corona), the predicted currents exceeded measured ones by a factor of more than 2 for very narrow pole spacings.

- The explanation proposed in this paper relates to a local discrepancy between the area in which corona occurs 
in reality (lower conductor side during rain) and the conductor region subject to an increased space charge enhanced field (the side facing the opposite pole). This hypothesis was substantiated through the comparison with an experimental case in which disturbances were positioned around the entire conductor circumference.

- It seems plausible that all numerical methods that do not account for a location bias with regard to ion injection on the conductor surface would suffer from the same issue (for foul weather CL).

\section{B. Hybrid AC/DC Corona}

- Controlled laboratory studies confirmed that a coronating $\mathrm{AC}$ conductor emits a net DC ion current if a DC field is superimposed. The result is a bipolar space charge environment, leading to a mutual enhancement of each conductors' corona activity, similar to the behavior in a bipolar HVDC system.

- The assumption made in certain previous publications that the hybrid ion flow problem can be solved by assuming a grounded AC conductor clearly does not hold in general. In particular in foul weather, when $\mathrm{AC}$ and DC conductors will both produce significant amounts of corona, large deviations from this assumption can be expected.

- A narrow separation distance between $\mathrm{AC}$ and $\mathrm{DC}$ conductors increases the influence that $\mathrm{AC}$ corona has on the ion flow coupling.

- Full-scale outdoor test data on the impact of AC corona on the conductor ion currents in hybrid AC/DC systems is very rare but the few data points that are available indicate a very large impact.

- Numerical methods in the literature that specifically model AC corona for the hybrid AC/DC ion flow problem have limited their comparison to ground level quantities. Extending model comparisons to conductor currents would be of very high interest, in particular for time-domain approaches.

\section{ACKNOWLEDGEMENTS}

The authors would like to thank Michiel Tavernier for the design of the artificial disturbances. The work was supported by the SNSF NRP 70 programme.

\section{REFERENCES}

[1] V. L. Chartier, S. H. Sarkinen, R. D. Stearns et al., "Investigation of Corona and Field Effects of AC/DC Hybrid Transmission Lines," IEEE Trans. Power App. Syst., vol. PAS-100, no. 1, pp. 72-80, 1981.

[2] Electric Power Research Institute, "Hybrid Transmission Corridor Study: Volume 2: Phase 2-Full-Scale Tests," 1991.

[3] U. Straumann and C. M. Franck, "Ion-Flow Field Calculations of AC / DC Hybrid Transmission Lines," IEEE Trans. Power Del., vol. 28, no. 1, pp. 294-302, jan 2013.

[4] B. Rusek, C. Neumann, S. Steevens et al., "Ohmic coupling between AC and DC circuits on hybrid overhead lines," in Cigre Auckland Symposium, Auckland, 2013.

[5] V. Popkov, The theory of bipolar corona on conductors. National Research Council Canada, Technical Translations, 1948.
[6] M. Sarma and W. Janischewskyj, "Analysis of Corona Losses on DC Transmission Lines Part II - Bipolar Lines," IEEE Trans. Power App. Syst., vol. PAS-88, no. 10, pp. 1476-1491, oct 1969.

[7] W. Janischewskyj and G. Gela, "Finite Element Solution for Electric Fields of Coronating DC Transmission Lines," IEEE Trans. Power App. Syst., vol. PAS-98, no. 3, 1979.

[8] M. Abdel-Salam and Z. Al-Hamouz, "A finite-element analysis of bipolar ionized field," Trans. Ind. Appl., vol. 31, no. 3, 1995.

[9] W. Li, B. Zhang, J. He et al., "Ion Flow Field Calculation of Multicircuit DC Transmission Lines," 2008 International Conference on High Voltage Engineering and Application, no. 3, pp. 16-19, nov 2008.

[10] Y. Zhen, X. Cui, T. Lu et al., "High Efficiency FEM Calculation of the Ionized Field Under HVDC," IEEE Trans. Magnetics, vol. 48, no. 2, 2012.

[11] W. Li, B. Zhang, R. Zeng et al., "Discussion on the Deutsch Assumption in the Calculation of Ion-Flow Field Under HVDC Bipolar Transmission Lines," IEEE Trans. Power Del., vol. 25, no. 4, pp. 2759-2767, 2010.

[12] T. Guillod, M. Pfeiffer, and C. M. Franck, "Improved Coupled Ion-Flow Field Calculation Method for AC/DC Hybrid Overhead Power Lines," IEEE Trans. Power Del., vol. 29, no. 6, pp. 2493-2501, dec 2014.

[13] T. D. Bracken, A. S. Capon, and D. V. Montgomery, "Ground Level Electric Fields and Ion Currents on the Celilo-Sylmar $400 \mathrm{kV}$ DC Intertie During Fair Weather," IEEE Trans. Power App. Syst., vol. PAS97, no. 2, pp. 370-378, 1978.

[14] R. D. Dallaire and P. S. Maruvada, "Corona Performance of a 450$\mathrm{kV}$ Bipolar DC Transmission Line Configuration," IEEE Trans. Power Del., vol. 2, no. 2, pp. 477-485, 1987.

[15] P. S. Maruvada, "Electric Field and Ion Current Environment of HVdc Transmission Lines: Comparison of Calculations and Measurements," IEEE Trans. Power Del., vol. 27, no. 1, pp. 401-410, 2012.

[16] N. Hylten-Cavallius, S. Annestrand, H. Witt et al., "Insulation Requirements, Corona Losses, and Corona Radio Interference for High-Voltage D-C Lines," IEEE Trans. Power App. Syst., vol. 83, no. 5, pp. 500-508, may 1964.

[17] B. Bailey, "Progress Report on BPA HV DC Test Line Radio Noise and Corona Loss," IEEE Trans. Power App. Syst., vol. PAS-86, no. 10, pp. 1141-1145, oct 1967.

[18] E. Gehrig, A. Peterson, C. Clark et al., "Bonneville Power Administration's 1100-kV Direct Current Test Project: II - Radio Interference and Corona Loss," IEEE Trans. Power App. Syst., vol. PAS-86, no. 3, pp. 278-290, mar 1967.

[19] F. Hirsch and E. Schafer, "Progress Report on the HVDC Test Line of the $400 \mathrm{kV}$-Forschungsgemeinschaft: Corona Losses and Radio Interference," IEEE Trans. Power App. Syst., vol. PAS-88, no. 7, pp. 1061-1069, jul 1969.

[20] G. B. Johnson, "Electric Fields and Ion Currents of a $400 \mathrm{kV}$ HVDC Test Line," IEEE Trans. Power App. Syst., vol. PAS-102, no. 8, pp. 2559-2568, aug 1983 .

[21] P. S. Maruvada, R. Dallaire, P. Heroux et al., "Long-Term Statistical Study of the Corona Electric Field and Ion-Current Performance of a 900-kV Bipolar HVDC Transmission Line Configuration," IEEE Trans. Power App. Syst., vol. PAS-103, no. 1, pp. 76-83, jan 1984.

[22] P. S. Maruvada, N. Trinh, R. D. Dallaire et al., "Corona Studies for Biploar HVDC Transmission at Voltages Between $600 \mathrm{kV}$ and 1200 kV Part 1: Long-Term Bipolar Line Studies," IEEE Trans. Power App. Syst., vol. PAS-100, no. 3, pp. 1453-1461, mar 1981.

[23] L. D. Anzivino, G. Gela, W. W. Guidi et al., "HVDC transmission line reference book," p. 684, 1993

[24] U. Corbellini and P. Pelacchi, "Corona losses in HVDC bipolar lines," IEEE Trans. Power Del., vol. 11, no. 3, pp. 1475-1481, jul 1996.

[25] B. A. Clairmont, G. B. Johnson, L. E. Zaffanella et al., "The effect of HVAC-HVDC line separation in a hybrid corridor," IEEE Trans. Power Del., vol. 4, no. 2, pp. 1338-1350, 1989.

[26] B. Sander, J. Lundquist, I. Gutman et al., "Conversion of AC multi- 
circuit lines to AC-DC hybrid lines with respect to the environmental impact," in Cigre Session, Paris, vol. B2, no. 105, 2014.

[27] S. Hedtke, M. Pfeiffer, C. M. Franck et al., "Audible noise of hybrid AC / DC overhead lines : Comparison of different prediction methods and conductor arrangements," in EPRI's High-Voltage Direct Current \& Flexible AC Transmission Systems Conference, vol. 1, 2015, pp. 1-8.

[28] M. Raghuveer, "Laboratory investigation of hybrid AC/DC transmission system corona performance," J. Electrostatics, vol. 22, no. 3, pp. 279288, sep 1989.

[29] M. Pfeiffer, J. Schmutz, C. M. Franck et al., "DC ion-currents in AC conductors in hybrid AC / DC transmission systems," in IET Conf. on AC/DC Power Transm., Birmingham, 2015.

[30] M. Beltle, M. Schühle, and S. Tenbohlen, "Influences of Direct Currents on Power Transformers Caused by AC-HVDC Interactions in Hybrid Grids," in International Symposium on High Voltage Engineering (ISH), Pilsen, Czech Republic, 2015.

[31] P. S. Maruvada and S. Drogi, "Field and ion interactions of hybrid AC/DC transmission lines," IEEE Trans. Power Del., vol. 3, no. 3, pp. 1165-1172, 1988.

[32] T. Zhao, S. A. A. Sebo, D. G. G. Kasten et al., "Calculation of single phase AC and monopolar DC hybrid corona effects," IEEE Trans. Power Del., vol. 11, no. 3, pp. 1454-1463, 1996.

[33] W. Li, B. Zhang, J. He et al., "Calculation of the ion flow field of AC-DC hybrid transmission lines," IET Gener. Transm. Distrib., vol. 3, no. 10, pp. 911-918, 2009.

[34] H. Yin, J. He, B. Zhang et al., "Finite Volume-Based Approach for the Hybrid Ion-Flow Field of UHVAC and UHVDC Transmission Lines in Parallel," IEEE Trans. Power Del., vol. 26, no. 4, pp. 2809-2820, 2011.

[35] X. Zhou, X. Cui, T. Lu et al., "A Time-Efficient Method for the Simulation of Ion Flow Field of the AC-DC Hybrid Transmission Lines," IEEE Trans. Magn., vol. 48, no. 2, pp. 731-734, 2012.

[36] B. Zhang, J. Mo, J. He et al., "A Time-Domain Approach of Ion Flow Field Around ACDC Hybrid Transmission Lines Based on Method of Characteristics," IEEE Transactions on Magnetics, vol. 53, no. 3, 2016.

[37] W. Li, B. Zhang, R. Zeng et al., "Study on the field effects under reduced-scale DC/AC hybrid transmission lines," IET Generation, Transmission \& Distribution, vol. 7, no. 7, pp. 717-723, 2013.

[38] M. Pfeiffer, T. Schultz, S. Hedtke et al., "Explaining the impact of conductor surface type on wet weather HVDC corona characteristics," J. Electrostatics, vol. 79, pp. 45-55, 2016.

[39] M. Akazaki, "Corona Phenomena from Water Drops on Smooth Conductors under High Direct Voltage," IEEE Trans. Power App. Syst., vol. 84, no. 1, pp. 1-8, jan 1965.

[40] S. Hedtke, M. Pfeiffer, A. Gaillard et al., "Effect of electrostatic induction and space charges on the audible corona noise of hybrid AC/DC transmission lines," in CIGRE-IEC Colloquium on EHV and UHV (AC \& DC), Montreal, 2016.

[41] M. Hara, S. Ishibe, and M. Akazaki, "Corona discharge and electrical charge on water drops dripping from DC transmission conductors - an experimental study in laboratory," J. Electrostatics, vol. 6, 1979.

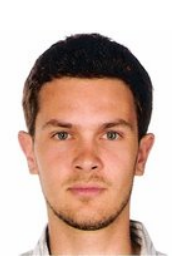

Martin Pfeiffer was born in Cotonou, Benin, in 1986. He received the M.Eng. degree in Engineering and Business Finance from University College London, UK, in 2008 and the M.Sc. degree in Energy Science and Technology from ETH Zurich, Switzerland, in 2012. In 2012, he joined the High Voltage Laboratory at ETH Zurich as a Ph.D. student. His main research topics are corona effects on HVDC overhead lines and their impact on potential hybrid $\mathrm{AC} / \mathrm{DC}$ transmission lines.

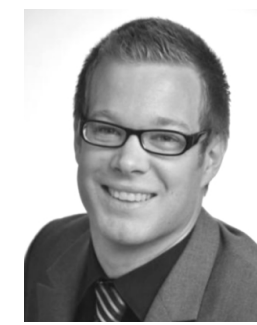

Sören Hedtke was born in 1988 in Germany and studied Electrical Engineering and Information Technology at the Technical University of Darmstadt (Germany) and the Grenoble Institute of Technology (France). Since he finished his Masters degree in 2014, he is with the High Voltage Laboratory at the Swiss Federal Institute of Technology (ETH Zurich). His current Ph.D. research focuses on hybrid AC/DC overhead lines with a special interest on the influence of coupling effects on corona phenomena as audible noise.

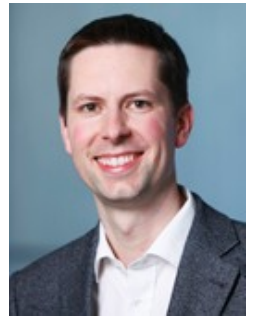

Christian M. Franck (M04-SM11) received a diploma in physics from the University of Kiel, Germany in 1999 and the Ph.D. degree in physics from the University of Greifswald, Germany in 2003. $\mathrm{He}$ was with the Swiss corporate research center of ABB from 2003-2009 as a Scientist and Group Leader for gas circuit breakers and high-voltage systems. Currently, he is Professor for High Voltage Technology at the Swiss Federal Institute of Technology (ETH) in Zurich, Switzerland. 